\title{
Evolution of intermediate excitons in fluid argon and krypton
}

\author{
P. Laporte and J. L. Subtil \\ Equipe de Spectroscopie de Centre National de la Recherche Scientifique (LA 171), F-42023 Saint-Etienne Cedex, France \\ R. Reininger and V. Saile \\ Hamburger Synchrotronstrahlungslabor (HASYLAB) at DESY, D-2000 Hamburg 52, Federal Republic of Germany
}

\author{
S. Bernstorff \\ Berliner Elektronenspeicherring Gesellschaft für Synchrotronstrahlung mbH, (BESSY), Lentzeallee 100, D-1000 Berlin 33, \\ Federal Republic of Germany \\ I. T. Steinberger \\ Racah Institute of Physics, The Hebrew University of Jerusalem, Jerusalem, Israel
}

(Received 8 September 1986)

\begin{abstract}
Reflectivity spectra of fluid argon and krypton are reported for number densities ranging from $\rho \sim 10^{21} \mathrm{~cm}^{-3}$ to that of the triple-point liquid, complemented by spectra of solid samples near the triple point. Dispersion analysis by a decomposition into Lorentzians of the complex dielectric constant spectra (yielding reflectivities that fit the experimental values) revealed the existence of "extra" absorption bands in the immediate vicinity of the ${ }^{3} P_{1}$ and ${ }^{1} P_{1}$ atomic resonance lines, growing very rapidly with density; these bands were identified as corresponding to the $n=1 \Gamma\left(\frac{3}{2}\right)$ and $n^{\prime}=1$ $\Gamma\left(\frac{1}{2}\right)$ intermediate excitons in the solid. Comparing the evolution of the $n=1$ exciton in fluid krypton with density fluctuations leads to the conclusion that for the creation of this exciton a momentary minimum local density of $10^{22} \mathrm{~cm}^{-3}$ is needed within a certain small volume $\left(1.2 \times 10^{-21} \mathrm{~cm}^{3}\right)$. The results are also discussed in terms of a recent theory on localization in topologically disordered systems. Results on the changes in characteristic parameters, like line separations, widths, and oscillator strengths are presented.
\end{abstract}

\section{INTRODUCTION}

Wannier excitons in a pure liquid ${ }^{1}$ are entities that have no atomic (or molecular) parentage but a rather close relationship with Wannier excitons in the corresponding solid. Such excitons are well known from studies of liquid xenon ${ }^{2,3}$ and dense fluid xenon ${ }^{4}$ and were recently reported for liquid krypton ${ }^{5}$ as well. This paper, however, deals with absorption bands in liquids that are very close to an atomic (or molecular) line and states conditions for regarding such bands as excitons.

It has been shown ${ }^{4,6}$ that in fluid xenon there exist "extra" absorption bands very near to broadened and shifted atomic lines; these bands appear only above a certain characteristic density and evolve as a function of density quite differently from the shifting and broadening of their "atomic" neighbors. One particular xenon band, just below the band corresponding to the first xenon resonance line $6 s^{3} P_{1}$ was studied in detail: ${ }^{6}$ Its amplitude could be correlated with density fluctuations. This band was identified as the $n=1$ "intermediate" exciton; ${ }^{7}$ the correlation with density fluctuations was interpreted as an indication that for the appearance of an exciton within the fluid medium a certain minimum local density is needed. Such an enhanced local density would be necessary to ensure the delocalization of the excitation. The only substances besides xenon reported to exhibit distinct excitonic bands very near to broadened and shifted atomic (or, for that matter molecular) lines were fluid krypton ${ }^{8,9}$ and fluid argon. ${ }^{10}$ However, the reflectivity spectra indicating the formation of excitons in these fluids were not analyzed to obtain the optical constants as a function of photon energy and therefore no quantitative deductions could yet be made. A systematic study and analysis of the vacuumultraviolet optical spectra of these substances is needed not only to check the validity of deductions for xenon, but also in view of the fact that the gas-phase and solid-phase spectra of rare gas become more similar to each other the lighter the rare-gas atom: ${ }^{11}$ Thus the task to identify intermediate excitons as separate entities in the fluid becomes more intriguing the lighter the atom. In this paper reflectivity spectra in krypton and argon are presented for a wide range of fluid densities, starting with the gas near atmospheric pressure and up to the liquid near the triple point. Near-triple-point spectra of the respective solids are also presented. Dispersion analysis of the reflectivity spectra yields decomposition into Lorentzians, making it possible to follow the changes in the band parameters with density including those identified as excitons related to the "intermediate excitons" $n=1 \Gamma\left(\frac{3}{2}\right)$ and $n^{\prime}=1$ $\Gamma\left(\frac{1}{2}\right)$ in the solids. We note that the absorption peak of such an exciton is near the position of the $n p^{5}(n+1) s^{3} P_{1}$ or $n p^{5}(n+1) s{ }^{1} P_{1}$ atomic absorption line of the respective gas, and somewhat shifted from the predictions of a sim- 
ple hydrogenlike series; the Bohr radius is of the order of the interatomic distances in the solid.

The identification of "intermediate excitons" in liquid xenon as such is based to a large extent on continuity considerations: extrapolating the variation with density (achieved by temperature changes) of the respective exciton peak positions in the solid to the density of the triplepoint liquid furnishes a value that coincides (to a very good approximation) with that of the peak actually observed in the liquid. ${ }^{12,13}$ Band gaps and exciton binding energies $3,8,12,13$ also change in accord with the density change upon the solid-liquid phase transition. Measuring the energy $V_{0}$ of the conduction-band electron in rare-gas fluids ${ }^{14}$ and in methane ${ }^{15}$ and extrapolating it to the density of the triple-point solid again leads to values in accord with experiment. It seems that in rare gases and similar substances the phase transition influences the values of various energy parameters primarily through the density change involved. Extending the range of measurements to include the near-triple-point solids of krypton and argon enables us to make further tests on this point. However, the detailed dispersion analysis also furnishes answers to the question, whether there may exist other exciton parameters that change substantially upon the liquid-solid phase transition. Band widths, the amount of the spinorbit splitting, and electron-hole exchange interaction causing separation of the $n=1$ and $n^{\prime}=1$ intermediate excitons, ${ }^{16}$ central-cell corrections, ${ }^{17}$ and effective masses fall in this category of study. A related subject studied and discussed concerns the relative values of the oscillator strengths of the first two resonance lines in the gas as compared with those of their counterparts in the highdensity liquid or in the solid. Two predictions ${ }^{16,18}$ gave different results on this point; experimental results on the

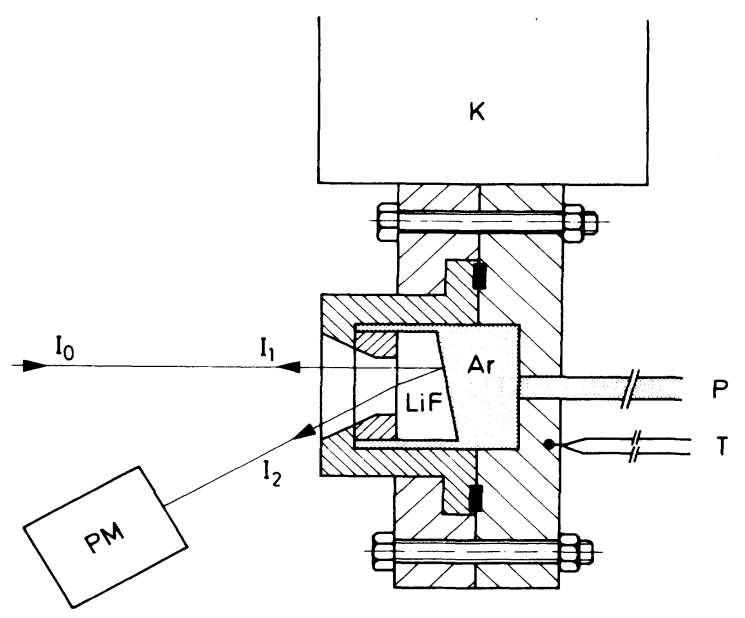

FIG. 1. Schematic representation of the cell used at HASYLAB indicating the beam positions. $K$ : cryostat; $P$ : pressure connection; $T$ : temperature control; LiF: wedged lithium fluoride window; Ar: gaseous, liquid, or solid argon (or krypton); $I_{0}$ : incident monochromatic light, monitored by a photomultiplier (not shown); $I_{1}$ : light reflected on the front side of the $\mathrm{LiF}$ window and not detected by the photomultiplier (PM); $I_{2}$ : light reflected from the $\mathrm{LiF} / \mathrm{Ar}$ or $\mathrm{LiF} / \mathrm{Kr}$ interface. transmission spectra of thin rare-gas films ${ }^{19}$ were found to be at considerable variances with theoretical predictions. It should be noted that the ratio of the oscillator strengths is rather sensitive to the choice of the wave functions involved.

Recently, Logan and Wolynes ${ }^{20}$ published a selfconsistent theory on localization of excitations applicable to gases and liquids. The effect of the topological disorder of this system is tackled by the methods of AbouChacra, Anderson, and Thouless. ${ }^{21}$ They start by assuming the states to be localized and mutually interacting via a classical interaction potential and determine the critical value of the strength of the transfer-matrix element (represented by a dimensionless quantity $U$ ), needed to destroy the localization. The functional dependence of $U$ on the normalized density was calculated for various values of $N$, where $V(r)$ varies like $r^{-N}, V(r)$ determining the excitation transfer energy to a distance $r$. Logan and Wolynes calculated $U$ versus normalized density $\rho^{*}$ with different approximations; as intuitively expected, the critical value of $U, U_{c}$, is in all cases a monotonically decreasing function of $\rho^{*}$. We note that the localized states in the theory discussed correspond closely with the atomic excitations and the delocalized ones with excitons.

\section{EXPERIMENT}

All experiments were performed using stainless-steel sample cells that could be evacuated down to $10^{-6} \mathrm{~Pa}$ and could withstand pressures up to $10^{7} \mathrm{~Pa}$. The argon experiments and those on solid krypton were performed at the Hamburger Synchrotronstralungslabor, Hamburg (HASYLAB) with synchrotron radiation using the Honormi experimental station. ${ }^{22}$ In these experiments a LiF window was used. Most of the krypton spectra were obtained in Saint-Etienne, using a high performance VUV photometric setup. ${ }^{23}$ Since the primary interest in this work was the evolution of excitons near the first two atomic resonance lines and for $\mathrm{Kr}$ these are sufficiently remote from the $\mathrm{MgF}_{2}$ cutoff, this material was chosen for the window, because of its better optical and mechanical properties. Gases of nominal purity of at least 99.9996\% supplied by L'Air Liquide were used, without further purification. The stainless-steel gas-handling system was baked and evacuated to $10^{-6} \mathrm{~Pa}$ before each set of measurements. The thermodynamic path of the experimental series avoided the coexistence curve; this was achieved by applying pressures larger than the saturated vapor pressure at the given temperature. The reflectivity measurements were taken at near-normal incidence from the window-sample interface; we avoided recording light reflected from the front window surface by having the two window surfaces polished at an angle of $5^{\circ}$ between them. Figure 1 shows the cell used at HASYLAB. The spectral resolution was $3 \mathrm{meV}$ at HASYLAB and $5 \mathrm{meV}$ in Saint-Etienne.

\section{RESULTS}

Figure 2 shows a set of reflectivity spectra for $\mathrm{Ar} / \mathrm{LiF}$ interfaces, with the argon number density increasing from $\rho=0.425 \times 10^{21} \mathrm{~cm}^{-3}$ [spectrum (a), gas] to $24.8 \times 10^{21}$ 


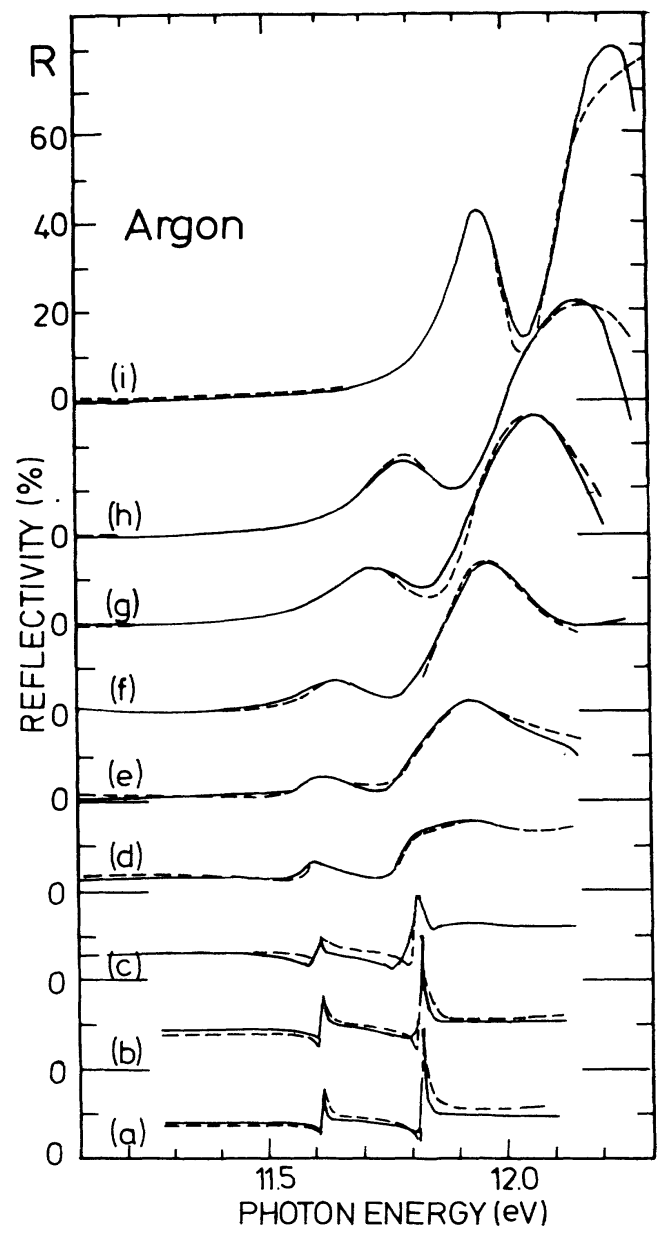

FIG. 2. Experimental (solid lines) and fitted (dashed lines) (see text) reflectivities of $\mathrm{Ar} / \mathrm{LiF}$ interfaces as a function of photon energy at various temperatures $(\mathrm{K})$ and number densities $\left(10^{21} \mathrm{~cm}^{-3}\right.$ ): (a) 147.3, 0.425; (b) 147.3, 0.814; (c) 147.7, 2.5; (d) $163.5,6.29$; (e) $155.3,9.9$; (f) $140.1,14.6$; (g) $111.8,18.5$; (h) 86.8 , 21.2; (i) (solid) 78.4, 24.8. The high-energy end of each spectrum is about $0.3 \mathrm{eV}$ below the $\mathrm{LiF}$ cutoff at the temperature of the measurement.

$\mathrm{cm}^{-3}$ [spectrum (i), solid]. The high-energy end of each spectrum is near the LiF absorption cutoff at the appropriate temperature. ${ }^{24}$ Because of the proximity of the cutoff, the reflectivity values of denser samples, at the decreasing flank of the higher-energy peaks, are associated with a larger experimental error, the worst case being that of solid argon [spectrum (i)] where only part of this peak falls within the experimentally accessible region.

The only features seen at the two lowest densities [Fig. 2 , spectra (a) and (b)] are the considerably broadened resonance lines $3 p^{5} 4 s^{3} P_{1}$ and $3 p^{5} 4 s^{1} P_{1}$. In Fig. 2, spectra (c) and $(d)$, one can also discern a further broad band at about $11.9 \mathrm{eV}$, i.e., on the blue side of the second resonance line. A less clear feature in the same graph is yet another rather broad band, having its center roughly coinciding with that of the first resonance line. With increasing density the broad "extra" bands grow much faster than the reso- nance lines, so that at $\rho=14.6 \times 10^{21} \mathrm{~cm}^{-3}$ [Fig. 2, spectrum (f)] the resonance lines are no more discernible.

The dashed lines on the spectra (a)-(i) of Fig. 2 are calculated reflectivities fitted to the experimental ones by means of a dispersion analysis, assuming that the absorption spectrum is determined by a set of Lorentzian oscillators. $^{25}$ In other words, we used the formulas for the complex dielectric constant $\hat{\epsilon}=\epsilon_{1}+i \epsilon_{2}$ :

$$
\hat{\epsilon}=\epsilon_{r}+\frac{4 \pi e^{2} \rho}{m} \sum_{j} \frac{f_{j}}{\left(\omega_{j}^{2}-\omega^{2}\right)-i \omega \gamma_{j}},
$$

the real quantity $\epsilon_{r}$ representing the contribution of peaks at frequencies above the experimental range, $e$ is the charge, and $m$ the mass of an electron, $\rho$ the number density of the atoms, and $f_{j}$ the oscillator strength of the $j$ th oscillator having the transition angular frequency $\omega_{j}$ and half-width $\gamma_{j}$. The fitted reflectivities $R$ were calculated from Eq. (4) after solving the two equations (2) and (3) for the complex refractive index $\hat{n}=n+i k$ :

$$
\begin{aligned}
& n^{2}-k^{2}=\epsilon_{1}, \\
& 2 n k=\epsilon_{2}, \\
& R=\left|\frac{\hat{n}-n^{\prime}}{\hat{n}+n^{\prime}}\right|^{2},
\end{aligned}
$$

$n^{\prime}$ being (the photon energy and temperature-dependent)

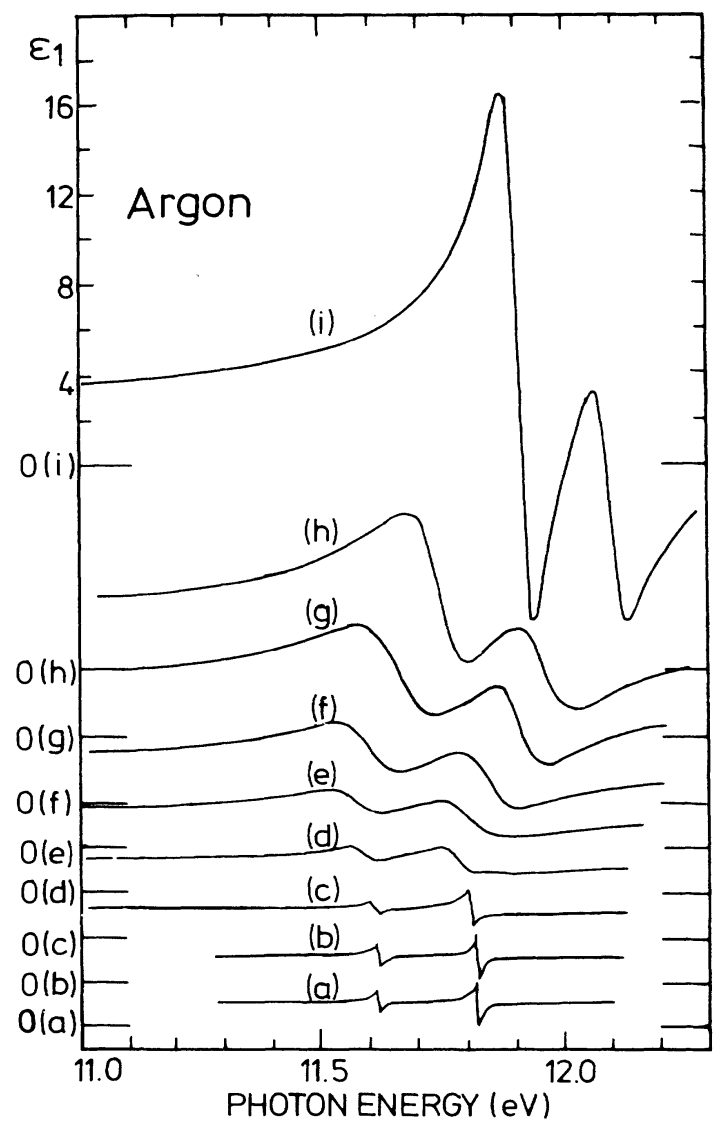

FIG. 3. Real part $\epsilon_{1}$ of the dielectric constant as a function of photon energy, obtained by means of the fits of Fig. 2. 
refractive index of the LiF substrate. ${ }^{24}$ The fits obtained were, in general, satisfactory with some exceptions: The fit in Fig. 2, spectrum (c), between 11.5 and $11.8 \mathrm{eV}$ could not be improved. This is probably due to the first appearance of the "extra" bands at this density. Furthermore the high-energy ends of the spectra of the denser samples could not be properly fitted, probably because of the lower accuracy of the experimental data near the LiF cutoff.

The thermodynamic conditions of the various runs (including some not appearing in Fig. 2) as well as the values of the parameters obtained by the fitting procedure are presented in Table I. It should be noted that the values of the background dielectric constant $\epsilon_{r}$ were not free parameters, but they were based on the optical constants measured on thin solid $\mathrm{Ar}$ films ${ }^{26}$ at $10 \mathrm{eV}$, using the Clausius-Mossotti relation to calculate $\epsilon_{r}$ at the various fluid densities. Figure 3 shows the real part $\epsilon_{1}$ of the dielectric constant and Fig. 4 the imaginary part $\epsilon_{2}$.

The positions of the band maxima $E_{i}$ as a function of the number density appear in Fig. 5(a). As stated above, it seems that the band at about $11.6 \mathrm{eV}$ is a composite one, but the components could not be resolved. The two bands at around $11.8 \mathrm{eV}$ are very close to each other and this makes their decomposition rather difficult especially in

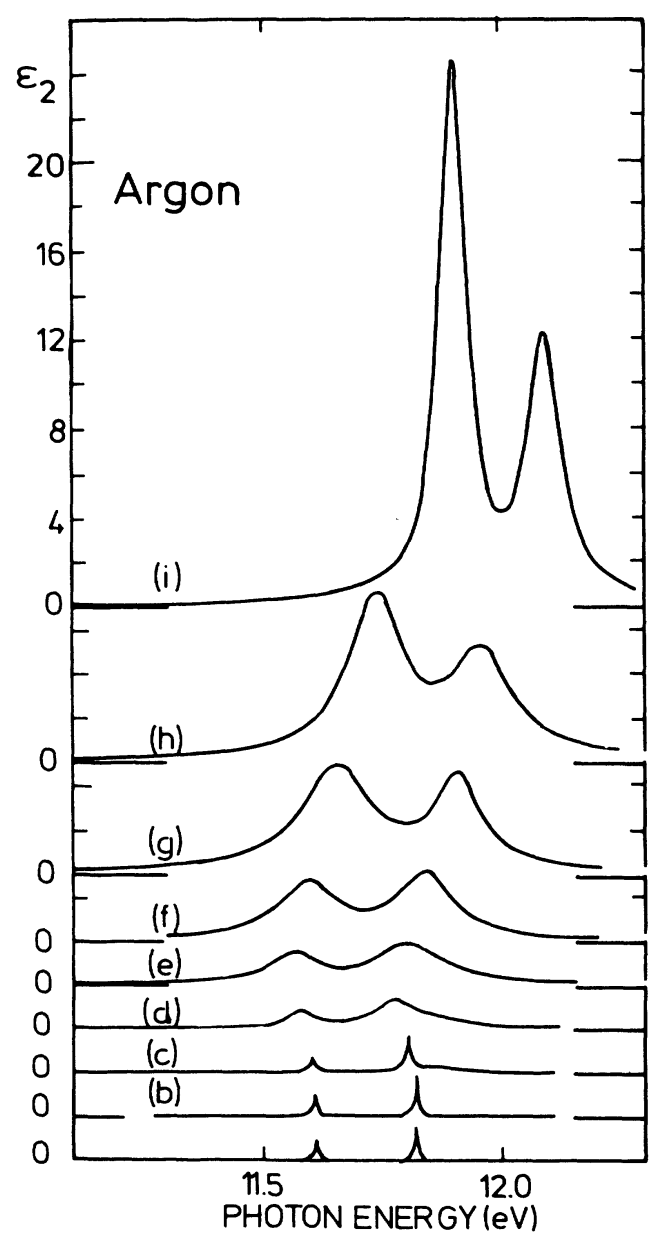

FIG. 4. Imaginary part $\epsilon_{2}$ of the dielectric constant as a function of photon energy, obtained by means of the fits of Fig. 2.

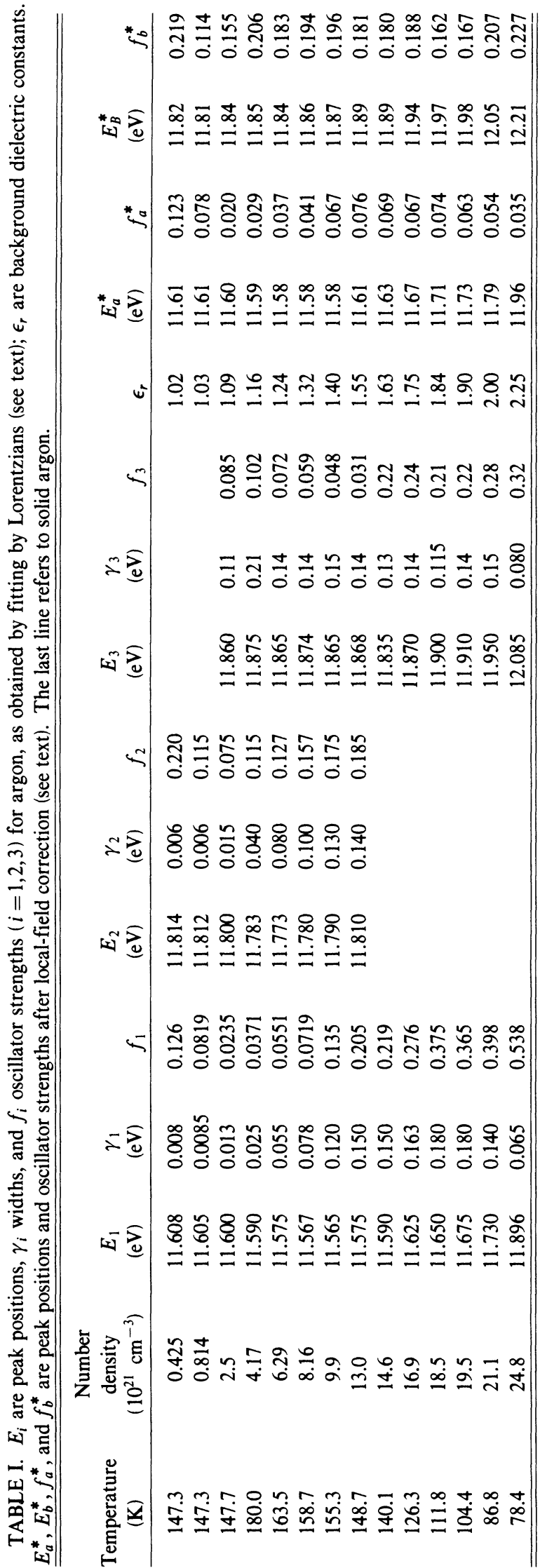




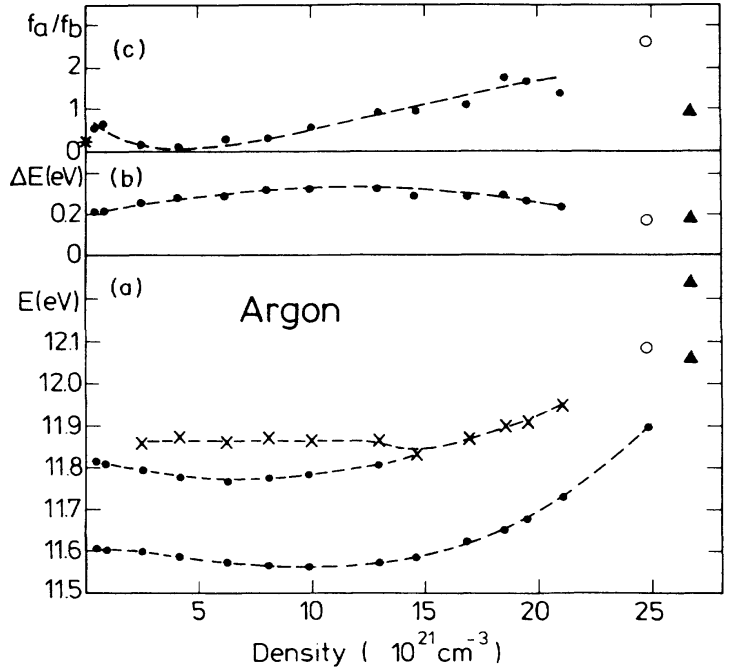

FIG. 5. Evolution with density of various parameters given by the analysis for argon. The solid triangles correspond to solid film experiments (Ref. 11); open circles, less reliable data due to the vicinity of the LiF cutoff (see text). (a) Peak positions $E_{i}$. As mentioned in the text, only the high-energy band could be separated on its atomic, $\bullet$, and excitonic, $\times$, contribution. (b) Energy separation $\Delta E$. (c) Oscillator strength ratios. * represents the ratio for the isolated atom.

the density range $(9.9-14.6) \times 10^{21} \mathrm{~cm}^{-3}$. For this region, the oscillator strengths $f_{2}, f_{3}$ and widths $\gamma_{2}, \gamma_{3}$ listed in Table I are also less reliable. Keeping these reservations in mind it is seen that with increasing density all bands shift at first to the red followed by a more pronounced blue shift. Figure 5(b) shows the energetic distance $\Delta E=E_{b}-E_{a}$ between the two systems, around $11.8 \mathrm{eV}$ and that around $11.6 \mathrm{eV}$, respectively, with $E_{a} \equiv E_{1}$ and $E_{b}$ being the arithmetic mean of $E_{2}$ and $E_{3}$. It is seen that $\Delta E$ increases gradually from about $0.2 \mathrm{eV}$ at the lowest densities to a very broad maximum $(\approx 0.26 \mathrm{eV})$ followed by a slow decrease to $0.19 \mathrm{eV}$ in the solid. In Fig. 5(c) we present the oscillator-strength ratio $f_{a} / f_{b}$ with $f_{a} \equiv f_{1}$ and $f_{b}=f_{2}+f_{3}$. An initial downward trend (from 0.57 down to $\approx 0.15$ ) is followed by a monotonous rise to 1.7 at the fluid density of $19.5 \times 10^{21} \mathrm{~cm}^{-3}$. For this graph, however, there is too much scatter among the points corresponding to the highest densities (one for the triple-point liquid and two for the solid at different temperatures) preventing clear determination of the final trend of the line. It should be stressed again that the experimental points referring to the lowest points are dubious because of the non-Lorentzian line shape ${ }^{27,28}$ and the convolution problems caused by the sharpness of the peaks, while the blue ends of the spectra of the densest samples are inaccurate because of the vicinity of the LiF cutoff.

Figure 6 shows the reflectivity spectrum of krypton at various densities. ${ }^{29}$ The spectra (a)-(g) in Fig. 6 were taken with a $\mathrm{MgF}_{2}$ window, (h) (solid $\mathrm{Kr}$ ) with a $\mathrm{LiF}$ win-

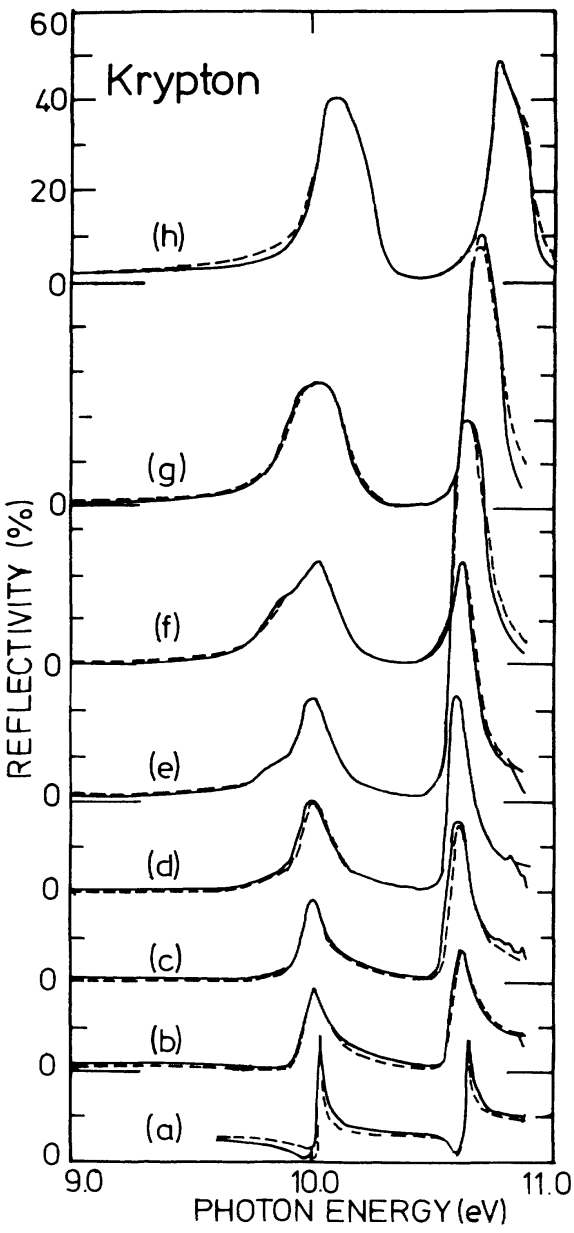

FIG. 6. Experimental (solid lines) and fitted (dashed lines) reflectivities as a function of photon energy. Spectra (a)-(g): $\mathrm{Kr} / \mathrm{MgF}_{2}$; spectrum $(\mathrm{h}): \mathrm{Kr} / \mathrm{LiF}$ interface. Temperatures $(\mathrm{K})$ and number densities $\left(10^{21} \mathrm{~cm}^{-3}\right)$ : (a) 230, 1.62; (b) 230, 5.13; (c) $228,7.32$; (d) $217,9.72$; (e) $199,12.15$; (f) $163,15.07$; (g) 122 , 17.44; (h) (solid) 113, 20.28

dow. At the low-energy side of the band at about $10 \mathrm{eV}$ (corresponding to the first resonance line $5 s^{3} P_{1}$ a further "extra" band can be seen in most spectra. This "extra" band appears already at a quite low density [Fig. 6, spectrum (c), $\rho=7.32 \times 10^{21} \mathrm{~cm}^{-3}$ ] and it grows rapidly with increasing density so that in the dense liquid of Fig. 6, spectrum (g), it encompasses the "atomic" band and only a single broad band is seen. No clear structure can be discerned at or around the second resonance li.ne $5 s^{1} P_{1}$, at about $10.65 \mathrm{eV}$, except for a pronounced broadening in Fig. 6, spectrum (b) $\left(\rho=5.13 \times 10^{21} \mathrm{~cm}^{-3}\right)$ compared with spectrum (a) $\left(\rho=1.62 \times 10^{21} \mathrm{~cm}^{-3}\right)$. It seems that a considerably broader peak is superimposed on the $5 s^{1} P_{1}$ peak between the two densities; a composite band results, with little change up to the triple point. The situation is analogous to that of the $4 s^{3} P_{1}$ peak in argon (see above). This point will be further discussed in Sec. IV.

The fitted curves (dashed lines) in Fig. 6 are much closer to the experimental results than those of Fig. 2. 


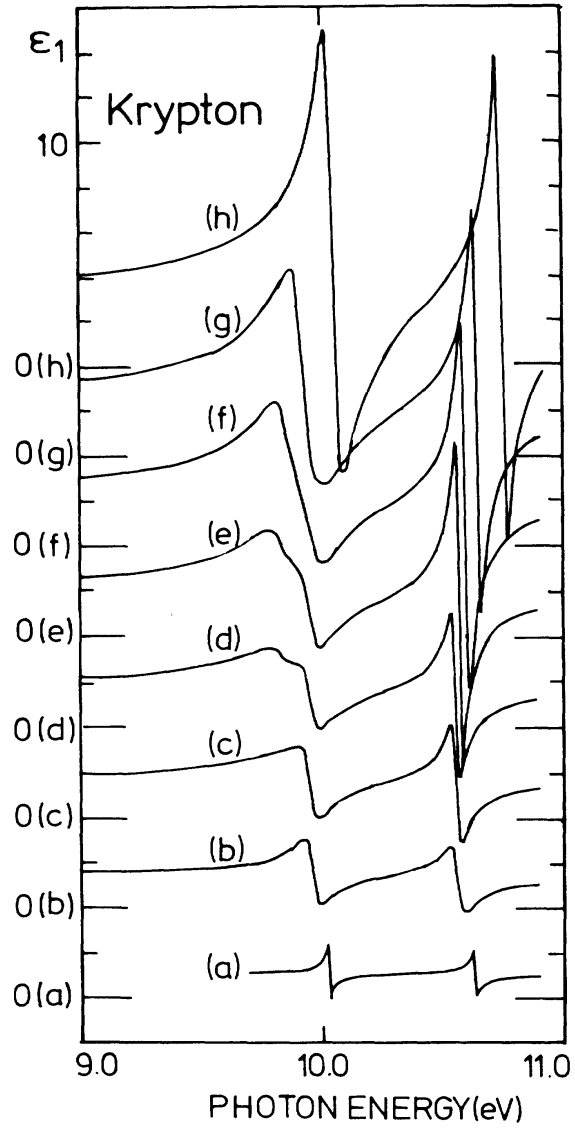

FIG. 7. Real part $\epsilon_{1}$ of the dielectric constant as a function of photon energy, obtained by means of the fits of Fig. 6 .

This is mainly due to the fact that the studied features of the krypton spectra are well separated from the absorption edge of the $\mathrm{MgF}_{2}$ window material. The higher reliability of the spectra warranted improving the fitting procedure too, namely by considering the contributions of a further band as well, beyond the high-energy limit of the experimental spectra. With this band we tried to approximate the totality of effects of the various energy bands beyond the experimental region. The $\epsilon_{r}$ values were, in this case, taken to be equal to the square of the refractive index $n_{r}$ in the visible range. ${ }^{30}$ In cases when there were no experimental results on $n_{r}$, the Clausius-Mossotti relation was used. The sets of curves for $\epsilon_{1}$ and $\epsilon_{2}$ appear in Figs. 7 and 8 , respectively. ${ }^{31}$ It is seen in the $\epsilon_{2}$ spectra that the "extra" peak is larger than its "atomic" companion ${ }^{3} P_{1}$ already in Fig. 8, spectrum (f), i.e., at $\rho=15.1 \times 10^{21}$ $\mathrm{cm}^{-3}$. The changes in the other main band, around 10.6 $\mathrm{eV}$, are smaller, the main effect being the very prominent broadening and shift between spectra (a) and (b) of Fig. 8 . With a further increase of the density this peak keeps growing and instead of broadening it becomes narrower. The thermodynamic conditions and the fitting parameters appear in Table II.

Figure 9(a) shows the density dependence of the peak positions of the various bands; as in argon, all bands ex-

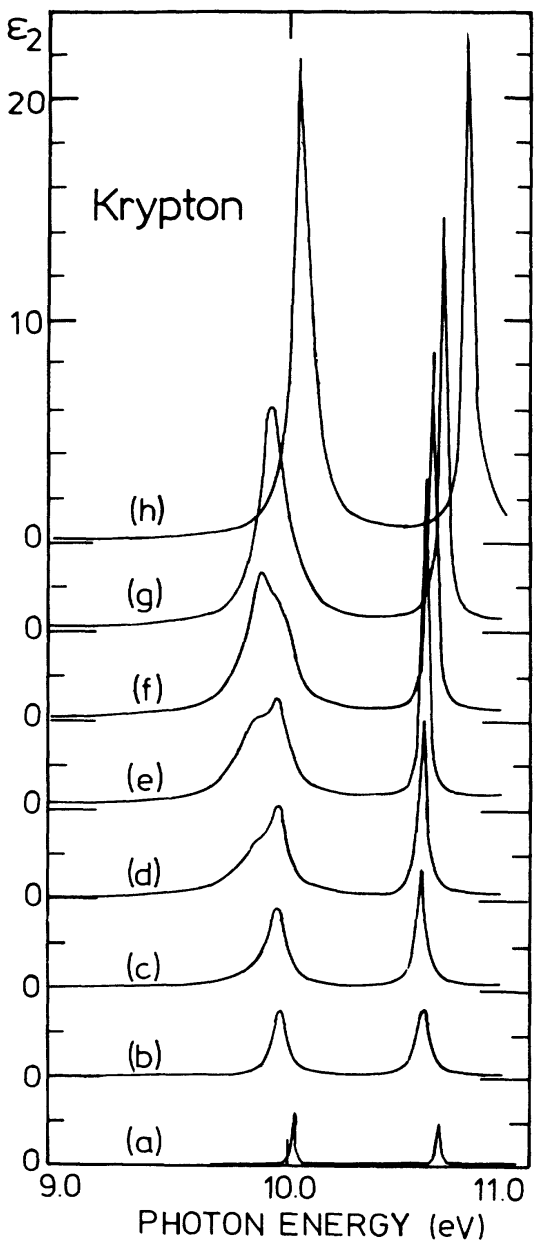

FIG. 8. Imaginary part $\epsilon_{2}$ of the dielectric constant as a function of photon energy, obtained by means of the fits of Fig. 6 .

hibit at first a moderate decrease followed by a rise. Figures 9 (b) and 9 (c) refer to the two band systems ( $a$ at $\approx 10$ $\mathrm{eV}$ and $b$ at $\approx 10.6 \mathrm{eV}$ ) and not to the individual bands. $E \equiv E_{b}-E_{a}$ is presented in Fig. 9(b); one discerns a monotonous rise starting from the lowest densities, in variance with Fig. 5(b). $f_{a} / f_{b}$ as a function of $\rho$ is shown in Fig. 9(c); its value increases from 0.7 to a maximum of 2.1 (at $\rho=1.38 \times 10^{22} \mathrm{~cm}^{-3}$ ) followed by a gradual drop. Comparing this with the corresponding graph for argon [Fig. 5(c)] again shows marked differences.

Figures 10 and 11 are shown to emphasize the differences between the "extra" band ("1") and the "atomic" band (" 2 ") near $10 \mathrm{eV} ; \gamma_{1} / \gamma_{2}$ can be seen to decrease monotonously (Fig. 10) with increasing $\rho$. This is due to the narrowing of the "extra" band and broadening of the atomic one. On the other hand, the oscillator strength fraction $f_{1} /\left(f_{1}+f_{2}\right)$ (Fig. 11) increases monotonously with increasing density, manifesting the growth of the "extra" band. Figure 11 will be discussed in detail in Sec. IV. 


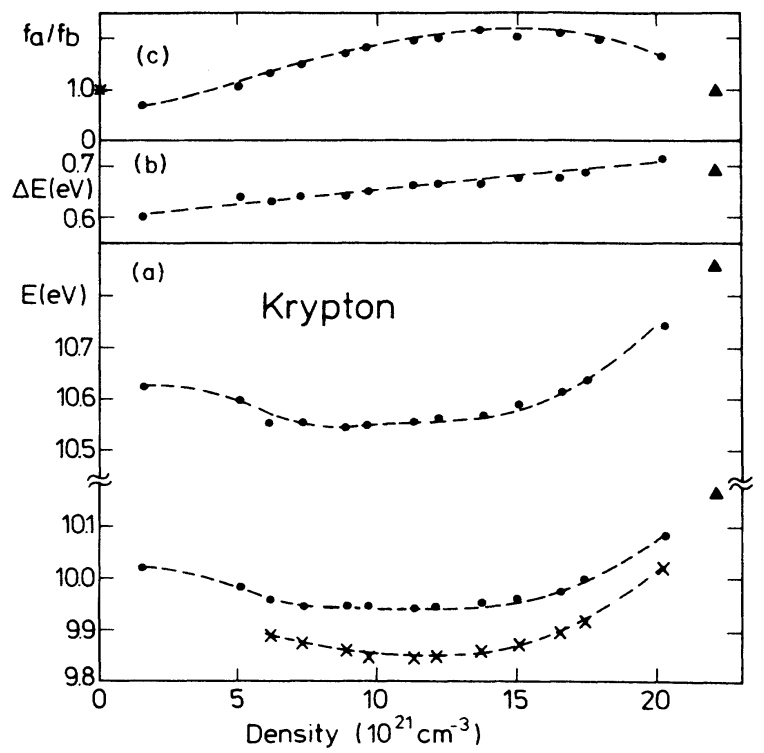

FIG. 9. Evolution with density of various parameters given by the analysis for krypton. The solid triangles correspond to solid films (Ref. 11). (a) Peak position $E_{i}$. To reproduce the line shape, two oscillators near $10 \mathrm{eV}$ were used for the solid as well. As mentioned in the text only the low-energy band could be separated on its atomic, $\bullet$, and excitonic, $\times$, contribution. (b) Energy separation $\Delta E$. (c) Oscillator strength ratios. represents the ratio for the isolated atom.

\section{DISCUSSION}

The results of this paper give a detailed description and analysis of the evolution of reflectivity spectra as a function of density, covering three thermodynamic phases. Besides the cases of $\mathrm{Ar}$ and $\mathrm{Kr}$ presented here only for $\mathrm{Xe}$ there exist similar, though less fully analyzed data. ${ }^{4,6}$ However, most theories for electronic states apply only for the two extrema of the density range studied: For

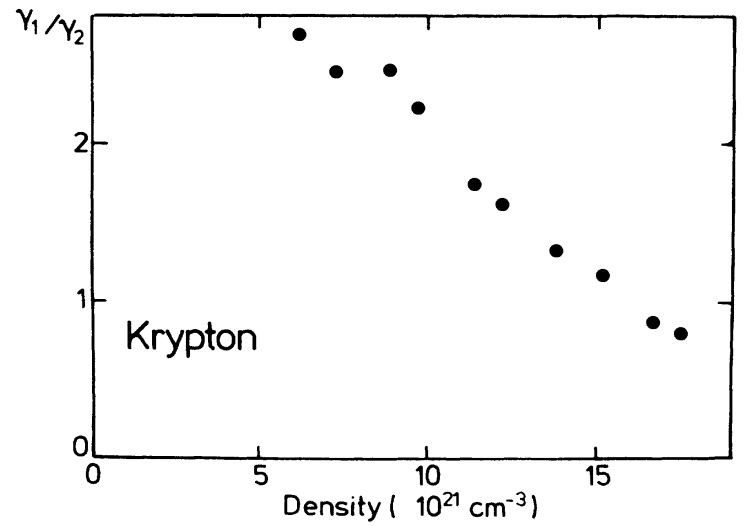

FIG. 10. Excitonic $\left[n=1, \Gamma\left(\frac{3}{2}\right)\right]$ to atomic $\left({ }^{3} P_{1}\right)$ half-width ratio $\gamma_{1} / \gamma_{2}$ vs density in the density range where both bands appear.

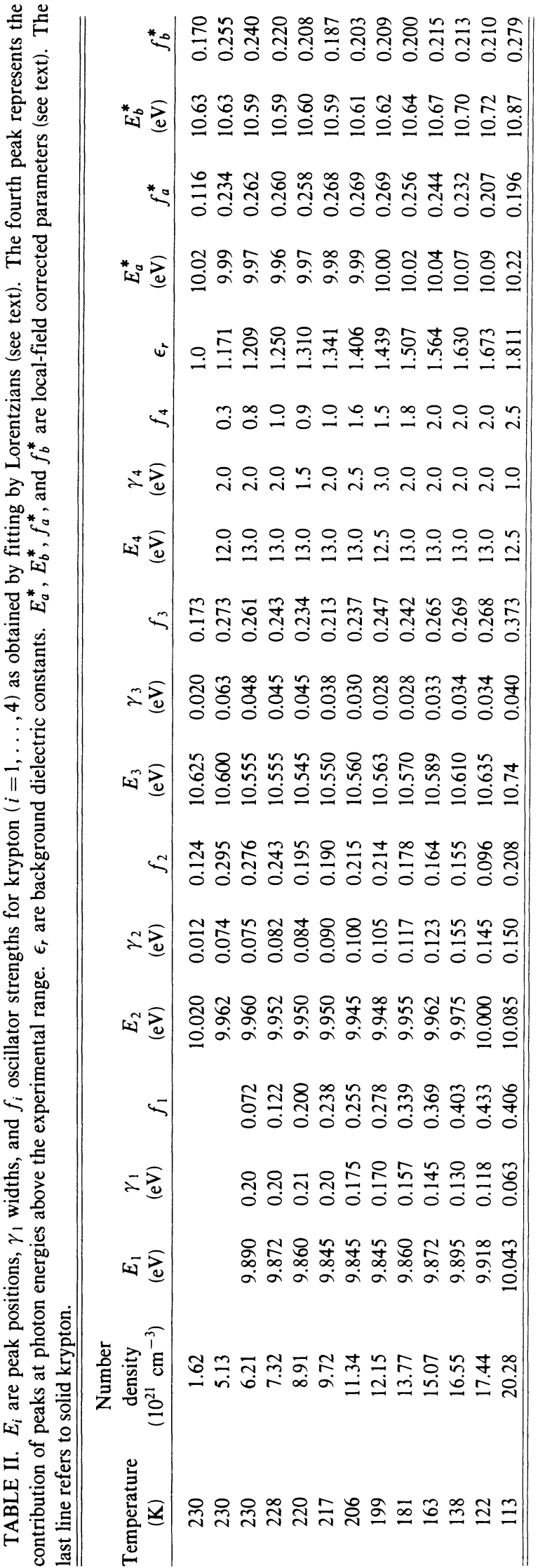




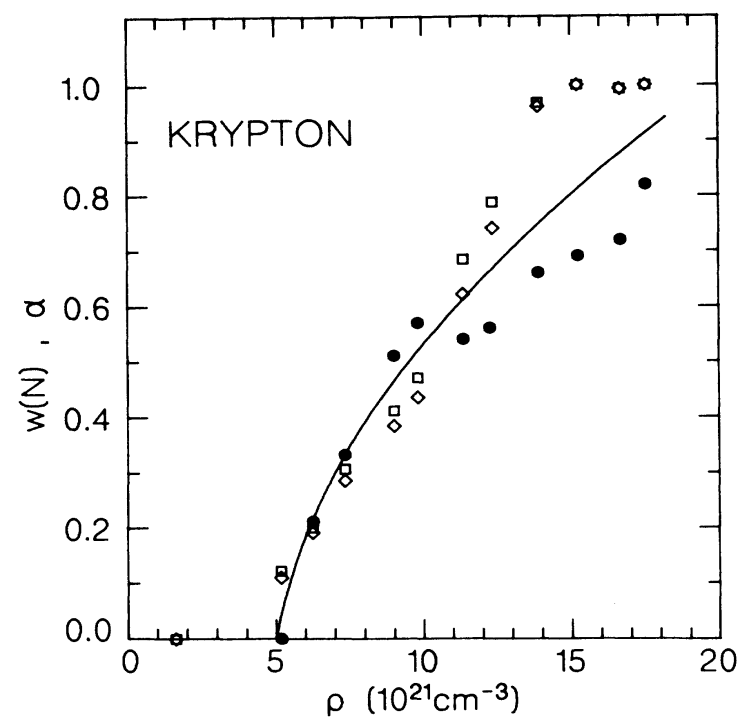

FIG. 11. Oscillator strength ratios $\alpha \equiv f_{1} /\left(f_{1}+f_{2}\right)$, $\bullet$, and probabilities $W\left(N_{0}\right)$ for $N_{0}=12$ atoms to be present in a volume $V_{0}$ of: $\square, 1.2 \times 10^{-21} \mathrm{~cm}^{3} ; \diamond, 1.15 \times 10^{-21} \mathrm{~cm}^{3}$ (see text) as a function of density.

krypton gas with $\rho<3 \times 10^{21} \mathrm{~cm}^{-3}$ it was shown ${ }^{28}$ that the absorption profiles, deduced from reflectivities, could serve to specify the effects of various forces, resonance, Van der Waals, and repulsive as well as the limits of applicability of the nearest-neighbor approximation of the quasistatic theory. At the other end of the range, exciton theories formulated for a crystalline solid can be applied. The central claim of this paper is that in most of the density ranges of the present experiments the absorption bands are dominated by excitons. The basis for this assignment will be presented below in some detail.

We recall that a series of reflectivity experiments in fluid xenon lead to two statements: (a) that Wannier excitons with no atomic parentage can be created in the system: ${ }^{2-4}$ (b) that very near to atomic lines (broadened and shifted by atomic and molecular interactions) the absorbed light may create entities different from the atomic excitations, namely "intermediate" excitons. ${ }^{7}$ In the spectral range discussed in this paper only "intermediate" excitons could be expected to appear.

\section{A. Intermediate excitons in fluid argon}

For argon the existence of the $n^{\prime}=1 \Gamma\left(\frac{1}{2}\right)$ intermediate exciton is indicated by the shape of the reflectivity versus density graph Fig. 2, spectrum (d) (see also Table I); its separation from the $3 p^{5} 4 s^{1} P_{1}$ atomic line is apparent in spectrum (c) of Fig. 2. A broadening of the bands indicates the creation of the $n=1 \Gamma\left(\frac{3}{2}\right)$ intermediate exciton. The distinction between exciton and atomic line could be, in principle, represented by excitation transfer integrals because of the partially Frenkel character of the intermediate excitons. ${ }^{7}$ Since the $n=1$ and $n^{\prime}=1$ excitons are red and blue shifted by different amounts with respect to the corresponding atomic transitions, the value and even the sign of these integrals should be both dependent on the type of the related atomic state.

At densities above $\rho=8.2 \times 10^{21} \mathrm{~cm}^{-3}$ [spectra (e) of Figs. 2 and 4 ] the excitonic peaks dominate the spectrum with no trace left of either atomic peak. Because of the proximity of the "extra" excitonic bands to their respective "atomic" counterpart and the considerable breadth of all bands (except at the lowest densities), it was not feasible to decompose the low-energy peak into the $n=1$ exciton and its ${ }^{3} P_{1}$ neighbor. Such a decomposition was possible for the other pair $\left(n^{\prime}=1\right.$ and $\left.{ }^{1} P_{1}\right)$ only within a limited density range $\left(2.5 \times 10^{21}<\rho<13 \times 10^{21} \mathrm{~cm}^{-3}\right)$. The variation of the peak positions, their mutual distance, and oscillator strength ratios as presented in Fig. 5 might serve for comparison with future theories. A further confirmation of the excitonic assignment is based on comparisons with spectra measured in the solid: In Figs. 5(a), $5(b)$, and 5(c) values of exciton parameters obtained from absorption spectra in thin argon films at about $10 \mathrm{~K}$ fall on the extrapolations of the appropriate curves for the fluid. The same is true for the $E$ and $\Delta E$ values obtained from reflectivity results on $78 \mathrm{~K}$ solids in the present experiments; the discrepancy of $f_{a} / f_{b}$ is due to the lower reliability of the data in the vicinity of the LiF cutoff (as indicated by open circles in Fig. 5).

\section{B. Intermediate excitons in fluid krypton}

The $n=1 \Gamma\left(\frac{3}{2}\right)$ intermediate exciton can be seen on the red side of the ${ }^{3} P_{1}$ atomic line starting with $\rho$ $=6.21 \times 10^{21} \mathrm{~cm}^{-3}$ [spectra (c) of Figs. 6 or 8 ]; its rapid growth with density is evident in Figs. 6, 8, and 11. It seems that the $n^{\prime}=1 \Gamma\left(\frac{1}{2}\right)$ intermediate exciton roughly coincides with the ${ }^{1} P_{1}$ atomic line and is dominant already in spectra (b) of Figs. 6 and $8 \quad\left(\rho=5.13 \times 10^{21}\right.$ $\mathrm{cm}^{-3}$ ). We note that for fluid xenon too, the $n^{\prime}=1$ exciton appears already at a density lower than the $n=1$ exciton. ${ }^{4}$ Its width changes in a rather unusual manner with density (Fig. 8 and Table II): when the density increases $(5-13) \times 10^{21} \mathrm{~cm}^{-3}$ and the temperature decreases from 230 to $122 \mathrm{~K}$, the half-width decreases from 0.063 to $0.028 \mathrm{eV}$, followed by a slight increase to $0.034 \mathrm{eV}$. No such behavior could be expected from a broadened and shifted atomic line. As in the case of argon, extrapolations of the graphs in Fig. 9 to solid density are in accord with actual measurements in the solid. It should be mentioned, however, that the reduced effective mass $m^{*}$ of the $\Gamma\left(\frac{3}{2}\right)$ exciton series behave differently: there is a discontinuity in $m^{*}$ upon the liquid-solid transition, ${ }^{5}$ as in the case of Xe. ${ }^{12}$ The marked differences between the excitonic ("extra") band and the atomic one were pointed out already in Sec. III: The width ratio $\gamma_{1} / \gamma_{2}$ decreases (Fig. 10) and the oscillator strength fraction $\alpha=f_{1} /\left(f_{1}+f_{2}\right)$ increases (Fig. 11) with increasing density, both features pointing to the quite different nature of the two bands. In fact, the narrowing and growth of band 1 when compared with band 2 are in full accord with the excitonic interpretation. For a quantitative discussion of Fig. 11, it will be assumed that excitons can be created only in certain environments, ${ }^{6}$ the nature of which will be specified below. Accordingly, in a first approximation $\alpha$ 
might be interpreted as the ratio of the number of atoms that are in such environments to the total number of atoms.

As in a work dealing with fluid xenon, ${ }^{6}$ the ratio $\alpha$ was compared with the probability $W\left(N_{0}\right)$ that in a small volume $V_{0}$ there should be at least $N_{0}$ atoms. Now, if $N_{A}$ is the average number of atoms in the volume, i.e., $N_{A}=V_{0} \rho_{A}$, where $\rho_{A}$ is the average density, then the mean-square fluctuation $\langle N\rangle^{2}$ is given by the thermodynamic formula ${ }^{32}$

$$
\langle N\rangle^{2}=\left(k_{B} T N_{A}^{2} / V_{0}\right) \kappa,
$$

$k_{B}$ being Boltzmann's constant, $T$ the absolute temperature, and $\kappa$ the isothermal compressibility. ${ }^{33,34}$ Then $W\left(N_{0}\right)$ is found from the area under the Gaussian

$W\left(N_{0}\right)=\frac{1}{\left(2 \pi\langle\Delta N\rangle^{2}\right)^{1 / 2}} \int_{N_{0}}^{\infty} \exp \left(-\frac{\left(N-N_{A}\right)^{2}}{2\langle\Delta N\rangle^{2}}\right) d N$.

Values of $V_{0}$ and $N_{0}$ were sought to obtain a fit of the $W\left(N_{0}\right)$ versus $\rho$ curve with $\alpha$ versus $\rho$. It is seen in Fig. 11 that a good correspondence at low and medium densities can be obtained for $V_{0}=1.2 \times 10^{-21} \mathrm{~cm}^{3}$ and $N_{0}=12$. Decreasing $V_{0}$ to $1.15 \times 10^{-21} \mathrm{~cm}^{3}$ spoils slightly the correspondence. The $V_{0}$ and $N_{0}$ values correspond to a local number density of $10^{22} \mathrm{~cm}^{-3}$; a similar analysis for fluid xenon yielded a local number density of $6.7 \times 10^{21} \mathrm{~cm}^{-3}$. This difference between the two materials is very reasonable, since for any given kind of interaction between neighboring atoms a higher number density is needed for the lighter krypton than for xenon. For example, the number densities for the triple-point liquids are $2.030 \times 10^{22} \mathrm{~cm}^{-3}(\mathrm{Kr})$ and $1.623 \times 10^{22} \mathrm{~cm}^{-3}(\mathrm{Xe}) ;^{35}$ the excitonic Bohr radii for the $n=1$ exciton (without central-cell correction) $0.26 \mathrm{~nm}$ for $\mathrm{Kr}$ and $0.37 \mathrm{~nm}$ for $\mathrm{Xe} ;{ }^{11}$ the "effective radii" of the ${ }^{3} P_{1}$ atomic states, 0.295 $\mathrm{nm}$ for $\mathrm{Kr}$ and $0.316 \mathrm{~nm}$ for Xe. ${ }^{36}$ The correlation found between $\alpha$ versus $\rho$ and $W\left(N_{0}\right)$ versus $\rho$ in these two substances gives quantitative support for the view that for the appearance of an exciton in the fluid the photon has to be absorbed at such an atom that has momentarily a sufficient number of sufficiently close neighbors to ensure delocalization of the excitation.

\section{Comparison with exciton theories}

Excitons in solidified rare gases have been the subject of theoretical efforts over many years. ${ }^{11,37}$ Calculation of excitation energies and oscillator strengths of excitons in such simple systems is a rather challenging task. Most existing theories can be divided into two categories: the solid-state physics and the atomic physics approach. The first is based on solid-state physics concepts with Wannier exciton series. This works well for excitons with principal quantum number $n \geq 2$. For the first excitons it fails and so-called "central-cell corrections" had to be introduced 17 taking into account the very small electron-hole separation of the first exciton states.

More sophisticated calculations especially for solid Ar are based on the integral-equation method developed by
Andreoni, Altarelli, and Bassani. ${ }^{16}$ While the exciton binding energies obtained by this theory are in very good agreement with the experimental data, ${ }^{19}$ significant discrepancies between the observed intensity ratio for the first two spin-orbit split excitons in the solid and the calculated ratio have been found. Both Kramers-Kronig analysis of reflection data ${ }^{38}$ and transmission data ${ }^{19}$ yielded experimentally an intensity ratio for $\operatorname{Ar} n=1$ to $n^{\prime}=1$ of $1: 1$ while theory predicts 1:6.5. Even on the basis of the experimental data presented here, it seems to be hardly possible to solve this discrepancy. The $f$ numbers obtained in the fit [see Fig. 5(c)] give a ratio of nearly 2:1 for the solid corresponding to a very low electron-hole exchange interaction ${ }^{16}$ ( $J J$-coupling limit). As stated above, the $f$ numbers presented are somewhat problematic, since for the second oscillator $\left(n^{\prime}=1\right.$ exciton) only the lowenergy part is experimentally accessible due to the cutoff of the LiF window, especially at high densities. Furthermore an appropriate description of the exciton line shape for excitons in the solid should be done on the basis of exciton-polaritons ${ }^{25}$ instead of simple Lorentzians.

There exist numerous attempts to describe excitons in terms of their related atomic transitions. For example, Resca and Resta ${ }^{39}$ developed a quantum defect theory for excitons in rare-gas solids. An atomic Schrödinger equation is modified by introducing effective masses for electron and hole and a dielectric screening of the Coulomb interaction between hole and electron. The band formation has been shown to develop continuously and smoothly with density ${ }^{40}$ and the dielectric constant as described by the Clausius-Mossotti relation is again a smooth function with density. On the other hand it has been shown for Xe (Ref. 6) and in the present work for $\mathrm{Kr}$ (and, qualitatively also for Ar) that excitons require for their existence a minimum local density in a small volume and are entities quite different from perturbed atomic Rydberg transitions. This is a rather strong argument against the atomic approach mentioned above. Other considerations $^{41}$ and experiments ${ }^{42}$ support this conclusion. Thus the quantum defect method in its present form does not seem to be the appropriate way to describe valence excitons in rare-gas solids.

The results presented in this paper along with those on $\mathrm{Xe}^{4,6}$ are suited for comparison with Logan's and Wolynes's ${ }^{20}$ recent theory on localization in fluids. While a full discussion of the implications of this fundamental theory is beyond the scope of the present paper, a few points should be stressed.

(i) The experiments show that in the fluid rare gases xenon, krypton, and argon, localized and delocalized states (excitons) can be readily distinguished spectroscopically, mainly by the energetic positions of the respective peaks. The trend of the widths and the oscillator strengths with density confirm the assignments. The clear distinctness of the two kinds of excited states is in full accord with the theory discussed, referring to two different types of states, with no continuous transition between them.

(ii) Logan and Wolynes represent the critical value $U_{c}$ of the transfer matrix element needed to destroy the localized states as a function of normalized density, $\rho^{*}=\rho \sigma^{3}, \sigma$ being a hard-core diameter. Taking for $\sigma$ the usual 
Lennard-Jones values and for $\rho$ the lowest density where the $n=1$ exciton appears, we find that for the three fluids the excitons appear at the same value of $\rho^{*}$, namely $0.30 \pm 0.01$.

(iii) In the fluids studied, localized and delocalized states coexist for broad density ranges. This coexistence should be possible if the two kinds of states exist in different local environments. The present authors suggest that characterization of the two kinds of environments in the framework of the theory will be a significant further test for it.

\section{Local-field corrections}

An evaluation of the data according to Eq. (1) bears an embiguity, i.e., the influence of local-field effects ${ }^{7}$ on energy positions and oscillator strengths derived. For excitations in a medium involving a well-localized state Lorentz-type local-field corrections are applicable. ${ }^{43-45}$ To the best knowledge of the present authors, only in very few cases $^{7,44}$ was the influence of such corrections discussed for excitons. In order to test the influence of local-field correction on our results such corrections were calculated utilizing a variant of the procedure suggested by Condell and Mandelberg ${ }^{45}$ for the gas phase. Considerations of the Lorentz local field for an isolated peak shows that the oscillator strength $f_{i}$ and peak energy $E_{i}$, obtained either by curve fitting or by Kramers-Kronig analysis, should be corrected to get $f_{1}^{*}$ and $E_{1}^{*}$ as follows:

$$
\begin{aligned}
& f_{i}^{*}=f_{i} \frac{9}{\left(\bar{n}_{0}^{2}+2\right)^{2}}, \\
& E_{i}^{*}=\left(E_{i}^{2}+E_{p}^{2} f_{i}^{*} \frac{\bar{n}_{0}^{2}+2}{9}\right)^{1 / 2}, \\
& E_{p}^{2}=\hbar^{2} \frac{4 \pi e^{2}}{m} \rho,
\end{aligned}
$$

$\bar{n}_{0}$ being the refractive index at the appropriate density, calculated as being the contribution of all oscillators except the $i$ th. For the estimation of $\bar{n}_{0}$ at the peak positions we used the data by Bideau-Méhu et al. ${ }^{46}$ on the dispersion of the refractive index, calculating $n_{0}$ at the approximate positions of the main peaks (104.82 and $106.66 \mathrm{~nm}$ for argon; 116.5 and $123.6 \mathrm{~nm}$ for krypton, see below) for normal conditions $\left(0^{\circ} \mathrm{C}, 760\right.$ Torr $)$ and deducing from this the value of $\bar{n}_{0}^{2}$ at the density in question by means of the Clausius-Mossotti formula. This formula is rigorous in the framework of the generalized semiclassical dispersion theory. ${ }^{45}$ Accordingly, close bands with strongly overlapping $\epsilon_{2}$ spectra (bands 2 and 3 for argon; bands 1 and 2 for krypton) cannot be treated separately.

Applying the above procedure for argon, we defined $f_{a}^{*} \equiv f_{1}^{*}$ and $E_{a}^{*} \equiv E_{1}^{*}[$ see Eqs. (7) and (8)] and

$$
\begin{aligned}
& f_{b}^{*}=\left(f_{2}+f_{3}\right) \frac{9}{\left(\bar{n}_{0}^{2}+2\right)^{2}}, \\
& E_{b}^{*}=\left(E_{b}^{2}+E_{p}^{2} f_{b}^{*} \frac{\bar{n}_{0}^{2}+2}{9}\right)^{1 / 2},
\end{aligned}
$$

$E_{b}$ being the average of the energies of the second and third peak. The parameters $f_{a}^{*}, f_{b}^{*}, E_{a}^{*}$, and $E_{b}^{*}$ appear in Table I. For krypton, a similar procedure, but with $E_{b}^{*} \equiv E_{3}$ and $f_{b}^{*}=f_{b}$ and the index " $a$ " referring to the combination of bands number 1 and 2, yielded the results listed as $f_{a}^{*}, f_{b}^{*}, E_{a}^{*}$, and $E_{b}^{*}$ in Table II.

Inspection of the tables reveals that in many cases the values of $f_{i}(i=1,2,3)$ increase considerably with density. It turns out that $f_{a}^{*}$ and $f_{b}^{*}$ do not show in any case the pronounced increases of $f_{i}$ with increasing density; in fact, introducing the local-field correction tends to stabilize the values of the oscillator strengths. The most important influence of the local-field correction in the context of this paper is a smaller variation of $f_{a} / f_{b}$. In fact with the correction $f_{a} / f_{b}$ approaches 0.2 and 0.6 instead of 1.8 and 1.6 for solid $\mathrm{Ar}$ and $\mathrm{Kr}$, respectively. On the other hand as it can be seen in Tables I and II that the effect on the energy positions is small.

While the corrections and consistency of local-field corrections is undoubted for localized excitations, its applicability for excitons is unclear. In particular, after demonstrating the delocalization of states in the fluid we refrain from a further discussion of the results obtained by the procedure Eqs. $\left(7^{\prime}\right)$ and $\left(8^{\prime}\right)$ for excitons in fluid rare gases. As Bergstresser and Rubloff pointed out ${ }^{44}$ the local-field correction applied here is not really correct but represents an upper limit for the influence of the polarizable medium. Further theoretical work on this question is highly desirable. An important step in this direction is a recent theory ${ }^{47}$ on the calculation of the polarizability of a liquid and how this polarization influences the electronic excitations of impurities.
'S. A. Rice and J. Jortner, J. Chem. Phys. 44, 4470 (1966).

${ }^{2}$ D. Beaglehole, Phys. Rev. Lett. 15, 551 (1965).

${ }^{3}$ I. T. Steinberger and U. Asaf, in Conduction in Low Mobility Materials, edited by N. Klein, D. S. Tannhauser, and M. Pollak (Taylor and Francis, London, 1971), p. 453.

${ }^{4}$ P. Laporte, J. L. Subtil, U. Asaf, I. T. Steinberger, and S. Wind, Phys. Rev. Lett. 45, 2138 (1980).

${ }^{5}$ P. Laporte, J. L. Subtil, R. Reininger, V. Saile, and I. T. Steinberger, Chem. Phys. Lett. 122, 525 (1985).

${ }^{6}$ P. Laporte and I. T. Steinberger, Phys. Rev. A 15, 2538 (1977).

${ }^{7}$ R. S. Knox, in Theory of Excitons (Academic, New York, 1963), Suppl. 5.
${ }^{8}$ U. Asaf and I. T. Steinberger, in Extended Abstracts, 5th International Conference on VUV Radiation Physics, Montpellier, France, 1977, edited by M. C. Castex, M. Pouey, and N. Pouey (CNRS, Meudon, 1977), Vol. 1, p. 205.

${ }^{9}$ R. Reininger, U. Asaf, I. T. Steinberger, P. Laporte, and V. Saile, Phys. Rev. B 26, 6294 (1982).

${ }^{10}$ S. Bernstorff, P. Laporte, R. Reininger, V. Saile, I. T. Steinberger, and J. L. Subtil, Vacuum Ultraviolet Radiation Physics, VUV 7, edited by A. Weinreb and A. Ron, VUV; Annals Israel Physical Society (Hilger, Bristol, 1983), Vol. 6, p. 270.

${ }^{11}$ B. Sonntag, in Rare Gas Solids, edited by M. L. Klein and J. A. Venables (Academic, London, 1977), Vol. 2, Chap. 17. 
${ }^{12}$ I. T. Steinberger and U. Asaf, Phys. Rev. B 8, 914 (1973).

${ }^{13}$ U. Asaf and I. T. Steinberger, Phys. Rev. B 10, 4464 (1974).

${ }^{14}$ R. Reininger, U. Asaf, I. T. Steinberger, and S. Basak, Phys. Rev. B 28, 4426 (1983); R. Reininger, U. Asaf, and I. T. Steinberger, Chem. Phys. Lett. 90, 287 (1982).

${ }^{15}$ U. Asaf, R. Reininger, and I. T. Steinberger, Chem. Phys. Lett. 100, 363 (1983).

16W. Andreoni, M. Altarelli, and F. Bassani, Phys. Rev. B 11, 2352 (1975).

17J. Hermanson and J. C. Philipps, Phys. Rev. 150, 652 (1966); J. Hermanson, ibid. 150, 660 (1966); U. Rössler and O. Schütz, Phys. Status Solidi B 56, 483 (1973).

${ }^{18}$ For example, L. Resca and R. Resta, Phys. Rev. B 21, 4889 (1980).

${ }^{19}$ V. Saile, Ph.D. thesis, Ludwig-Maximilians-Universität, Munich, 1976; V. Saile and E. E. Koch, Phys. Rev. B 21, 4892 (1980).

${ }^{20}$ D. E. Logan and P. G. Wolynes, Phys. Rev. B 29, 6560 (1984).

${ }^{21}$ R. Abou-Chacra, P. W. Anderson, and D. J. Thouless, J. Phys. C 6, 1734 (1973).

${ }^{22}$ V. Saile, P. Gürtler, E. E. Koch, A. Kozevnikov, M. Skibowski, and W. Steinmann, Appl. Opt. 15, 2559 (1976).

${ }^{23}$ P. Laporte, J. L. Subtil, M. Bon, and H. Damany, Appl. Opt. 20, 2133 (1981).

${ }^{24}$ P. Laporte, J. L. Subtil, M. Courbon, M. Bon, and L. Vincent, J. Opt. Soc. Am. 73, 1062 (1983).

${ }^{25}$ Lorentzian shape was assumed throughout even in domains where it is not the best approximation. For example, for the solid taking into account polariton and spatial dispersion effects was reported to give better fit to experiment than Lorentzians; see I. Ya. Fugol, O. N. Grigoraschenko, and E. V. Savchenko, Phys. Status Solidi B 111, 397 (1982).

${ }^{26}$ A. Harmsen, E. E. Koch, V. Saile, N. Schwentner, and M. Skibowski, in Vacuum Ultraviolet Radiation Physics, edited by E. E. Koch, R. Haensel, and C. Kunz (Pergamon Vieweg, Braunschweig, 1974), p. 339.

${ }^{27}$ M. C. Castex, M. Morlais, F. Spiegelmann, and J. P. Malrieu, J. Chem. Phys. 75, 5006 (1981); F. X. Gadea, F. Spiegelmann, M. C. Castex, and M. Morlais, ibid. 78, 7270 (1983).

28P. Laporte and H. Damany, J. Phys. (Paris) 40, 9 (1979).

${ }^{29}$ For results on reflection and absorption spectra at $\rho<3 \times 10^{21}$ $\mathrm{cm}^{-3}$ see Ref. 28 . Reflection spectra of liquid and solid krypton near the triple point and beyond $11.1 \mathrm{eV}$ are discussed in
Ref. 5.

${ }^{30}$ A. C. Sinnock, J. Phys. C 13, 2375 (1980)

${ }^{31}$ The consistency of the results was checked by comparing the presented $\epsilon_{1}$ and $\epsilon_{2}$ spectra, based on reflectivities of a liquidkrypton- $\mathrm{MgF}_{2}$ interface measured with a conventional source in Saint Etienne, with $\epsilon_{1}$ and $\epsilon_{2}$ spectra based on liquid-krypton-LiF interface reflectivities obtained with synchrotron radiation in Hamburg. The agreement between the two sets of spectra was very good in the common spectral range.

${ }^{32}$ T. L. Hill, Statistical Mechanics (McGraw-Hill, New York, 1956).

${ }^{33}$ Yu. P. Blagoi and V. A. Sorokin, Zh. Fiz. Khim. 44, 2745 (1970) [Russian J. Phys. Chem. 44, 1563 (1970)].

${ }^{34} \mathrm{~F}$. Theeuwes and R. J. Bearman, J. Chem. Thermodynamics 2, 171 (1970).

${ }^{35}$ G. A. Cook, Argon, Helium and the Rare Gases Vol. I. (Interscience, New York, 1961).

${ }^{36}$ H. A. Bethe and E. E. Salpeter, in Handbuch der Physik, edited by S. Flügge (Springer-Verlag, New York, 1957), Vol. XXXV.

${ }^{37}$ For a recent review see N. Schwentner, E. E. Koch, and J. Jortner, Electronic Excitations in Condensed Rare Gases (Springer-Verlag, Berlin, 1985).

${ }^{38}$ Numerous more recent Kramers-Kronig calculations by $\mathrm{V}$. Saile (unpublished) confirm the data quoted in Ref. 26.

${ }^{39}$ L. Resca and R. Resta, Phys. Rev. B 19, 1683 (1979).

${ }^{40}$ R. Reininger, U. Asaf, I. T. Steinberger, V. Saile, and P. Laporte, Phys. Rev. B 28, 3193 (1983).

${ }^{41}$ S. Bernstorff, V. Saile, and G. L. Findley, Chem. Phys. Lett. 125, 161 (1986)

${ }^{42}$ S. Bernstorff and V. Saile, Opt. Commun. 58, 181 (1986).

${ }^{43}$ F. Wooten, Optical Properties of Solids (Academic, New York, 1972).

${ }^{44}$ T. K. Bergstresser and G. W. Rubloff, Phys. Rev. Lett. 30, 794 (1973).

${ }^{45}$ W. J. Condell and H. I. Mandelberg, J. Opt. Soc. Am. 54, 973 (1964).

${ }^{46}$ A. Bideau-Méhu, Y. Guern, R. Abjean, and A. JohanninGilles, J. Quant. Spectrosc. Radiat. Transfer 25, 393 (1981).

${ }^{47}$ D. Chandler, K. Schweizer, and P. G. Wolynes, Phys. Rev. Lett. 49, 1100 (1982). 